\title{
ALGUNAS CARACTERISTICAS DE LOS ESTABLECIMIENTOS INDUSTRIALES, SEGUN EL ULTIMO CENSO INDUSTRIAL
}

$311: 711.2: 6$

por

Ignacio Ballester Ros

SUMARIO: I. INTRODUCCION.-II. EL NUMERO DE ESTABLECIMIENTOS INDUSTRIALES, LAS PERSONAS OCUPADAS Y LA POTENCIA INSTALADA: 1 . EL NÚMERO DE ESTABLECIMIENTOS INDUSTRIALES. 2. LAS PERSONAS OCUPADAS. 3. LA POTENCIA INSTALADA. 4. PERSonas ocupadas y POTENCIA INSTALADA POR ESTABLECIMIENTO.-III. EL TAMAÑO DE LOS ESTABLECIMIENTOS INDUSTRIALES.-IV. LA UBICACION PROVINCIAL DE LAS PRINCIPALES INDUSTRIAS, SEGUN RAMAS IDE LA ACTIVIDAD INDUSTRIAL.-V. APENDICE ESTADISTICO: 1. NGMERO DE ESTABLECIMIENTOS, PERSONAS OCUPADAS Y POTENCIA INSTALADA: A) Cifras absolutas. B) Porcentajes. C) Personas ocupadas y potencia instalada por establecimiento. 2. TAMAÑO DE LOS ESTABLECIMIENtos. PorcentaJes.

\section{INTRODUCCION}

La Ley de 8 de junio de 1957 encomendó al Instituto Nacional de Estadística la formación de los Censos Económicos y de un Plan Censal General, con la colaboración de los Ministerios interesados y de las Corporaciones locales. 
El citado Instituto, que ya realizaba desde el año 1857 los Censos Generales de la Población, ha realizado, al amparo de la precitada Ley, varios Censos de distinta naturaleza, entre los que cabe destacar los de Edificios de 1950 y 1970; de Viviendas de 1950, 1960 y 1970; el de Locales de 1970; los Agrarios de 1962 y 1972; el de Bibliotecas de 1960, y los de Establecimientos sanitarios y no sanitarios de 1964 y 1966, respectivamente.

En el mes de octubre de 1980 se ha iniciado la ejecución de los. Censos de Edificios y Locales, y en el mes de marzo del presente año ha comenzado igualmente la fase de recogida de datos para la formación de los Censos de la Población y Viviendas de 1981, juntamente con la renovación del Padrón Municipal de Habitantes. Y en 1982 se llevará a cabo el tercer Censo Agrario.

Sin embargo, el primer Censo que se realizó, tras la promulgación de la Ley de 1857, fue el Censo Industrial de 1958. Sólo veinte años. después, en 1978, se iniciaron los trabajos para la realización del segundo Censo Industrial, que había sido programado en dos fases. La primera, ya realizada, tenía por objeto conocer el número y distribución de las unidades industriales -empresas y establecimientos- por actividad económica, tamaño y áreas geográficas. La segunda fase, en ejecución, persigue el suministro de una amplia información, completa, homogénea y coherente, de la producción, de los principales bienes producidos, de la determinación del valor añadido y de la formación bruta de capital.

La primera fase comprendía a su vez dos etapas. La primera, de formación del directorio de establecimientos, trataba de conocer la estructura de los establecimientos industriales, mientras que la segunda estaba orientada esencialmente a las empresas. Los primeros. resultados del Censo Industrial, correspondiente a la primera etapa de la primera fase, han sido publicados recientemente.

Estos resultados se refieren a las actividades industriales comprendidas en las Divisiones 1 a 4 de la vigente Clasificación Nacional de Actividades Económicas, aprobada por el Real Decreto 2518/1974, de 1 de agosto. No comprenden los relativos a las actividades de producción, transporte y distribución de energía eléctrica, ni a los de construcción de edificios y obras públicas, que se han excluido de esta primera etapa, ya que, dada la naturaleza de las empresas respectivas, se ha considerado que la recogida de datos inicial debía estar orientada a las empresas más que a los establecimientos.

La información recogida, relativa a los establecimientos, hace referencia al número de establecimientos industriales existentes, las 
personas ocupadas - en la fecha de referencia del Censo y en el mes de máxima actividad- y la potencia instalada. Todo ello en relación con las características de actividad industrial, el tamaño de los establecimientos en función del número de personas ocupadas, la naturaleza jurídica de la empresa propietaria de los establecimientos, el tipo de establecimiento, el período de actividad y su ubicación según el tamaño de los Municipios.

Los citados resultados se han publicado, además de en un tomo de Resumen Nacional, en 50 monografías provinciales -además de otra para Ceuta y Melilla, conjuntamente- y en diez monografías relativas a las Comunidades Autónomas y Entes Preautonómicos, que comprenden más de una Provincia. En las monografías por Autonomías se insertan algunos datos por Provincias e igualmente en el Resumen Nacional, y las monografías provinciales contienen algunos datos por Municipios.

Considerando que esta información censal, de carácter estructural, tiene una importancia extraordinaria, dedicamos esta crónica al comentario de algunas características más significativas de la industria española, con especial referencia a las áreas autonómica y provincial, tomando como base el Resumen Nacional y las monografías relativas a las Autonomías.

\section{EL NUMERO DE ESTABLECIMIENTOS INDUSTRIALES, LAS PERSONAS OCUPADAS Y LA POTENCIA INSTALADA}

En el Apéndice Estadístico se insertan las tablas 1A) y 1B), en las que se presentan, respectivamente, las cifras absolutas correspondientes a la distribución por Autonomías y por Provincias, del número de establecimientos, de las personas ocupadas y de la potencia instalada. Igualmente se ha elaborado una tabla, 1C), en la que aparecen determinadas la relación personas ocupadas/establecimiento y la relación potencia instalada/establecimiento para las mismas áreas geográficas consideradas.

Analizaremos someramente dichas cifras, a fin de llegar a las conclusiones que permitan conocer el distinto peso de la estructura industrial de las Autonomías y de las Provincias.

\section{El NÚMERO DE ESTABLECIMIENTOS INDUSTRIALES}

El número de establecimientos industriales registrados en el Censo de referencia, con la cobertura descrita en la Introdución, ha sido 
de 195.162, con un promedio teórico de 3.903 por Provincia, equivalente al 2 por 100 del total.

Es evidente que la distribución de los establecimientos industriales no es uniforme sobre el área geográfica nacional. Por encima del promedio nacional se sitúan 16 Provincias, que, en conjunto, representan el 58,8 por 100 del total de establecimientos.

Destacan, en primer lugar, Barcelona, con más de la séptima parte del total, seguidas de Madrid, Valencia y Alicante. Entre estas cuatro Provincias absorben el 30 por 100 de los establecimientos. Las doce Provincias restantes, que superan el promedio nacional, ofrecen valores que oscilan entre el 2 y el 2,7 por 100. En algunas - las gallegas - no es significativo el número de establecimientos que registran, ya que se ha considerado cada abastecimiento de agua como un establecimiento.

En conjunto, las citadas 16 Provincias configuran las siguientes. zonas: a) Noroeste-Norte, con La Coruña, Lugo, Pontevedra, Asturias y Vizcaya. b) Zaragoza. c) Barcelona, Tarragona, Baleares, Alicante, Valencia y Murcia. d) Madrid, Toledo y Ciudad Real. e) Sevilla.

Con valores muy próximos al 2 por 100 se encuentran también: León, Guipúzcoa y Navarra, Gerona y Lérida, Badajoz y Granada, que amplian y complementan varias de las zonas enumeradas en el párrafo anterior.

Se perfila, por el contrario, una amplia zona de mínimo número. de establecimientos, formada por las Provincias de Avila, Palencia, Segovia, Soria, Zamora, Cuenca y Guadalajara, en ambas Castillas, a las que se unen Alava, Teruel, Huelva y Las Palmas.

Por Autonomías ocupa el primer lugar Cataluña que contiene la quinta parte de los establecimientos, a la que siguen Andalucía y el País Valenciano, con la octava parte cada una, Castilla-León, Galicia y Castilla-La Mancha, Madrid, el País Vasco, Aragón y, en último. término, Extremadura y Canarias, con los valores mínimos.

Es de subrayar que si incluimos en Castilla-La Mancha a la Provincia de Madrid, se obtiene la suma de dos componentes de igual valor, equivalente en conjunto al 13,6 por 100 del total de establecimientos, Región que se situaria en segundo lugar, después de $\mathrm{Ca}$ taluña.

Del examen de las cifras anteriores se desprende que, si bien el número de establecimientos es un indicador de la importancia relativa de la estructura industrial de las diferentes áreas consideradas, no lo es, en sí, plenamente definitivo, puesto que hay que tener muy 
en cuenta la población activa ocupada y el tamaño de los propios establecimientos, así como la potencia instalada, que examinamos a continuación.

\section{LAS PERSONAS OCUPADAS}

De la información disponible se deduce que en la fecha de referencia del Censo Industrial, las personas ocupadas en los establecimientos industriales alcanzaban la cifra de 2.811 .160 , con un promedio teórico de 4.649,68 personas por Provincia, equivalente igualmente al 2 por 100 del total.

Teniendo en cuenta este indicador se restringe ya el área territorial de las Provincias que presentan valores superiores al promedio nacional, que de 16 pasan a 12 , que en conjunto representan el 67,9 por 100 del total del personal empleado en los establecimientos industriales españoles.

Figuran, en primer lugar, Barcelona, con más de la quinta parte de la población activa industrial, y Madrid, con más del 10 por 100 . Les siguen a continuación Valencia, Vizcaya, Guipúzcoa y Asturias y Alicante. Entre las siete Provincias citadas disponen del 56,6 por 100 del personal ocupado en la industria. Otras cinco Provincias ofrecen valores que oscilan entre el 2 y el 2,7 por 100.

En conjunto, estas 12 Provincias configuran unas zonas mejor definidas:

a) Noroeste-Norte: Pontevedra, Asturias, Vizcaya, Guipúzcoa, Navarra y Zaragoza.

b) Oriental mediterránea: Barcelona, Alicante, Valencia y Murcia.

c) Madrid.

d) Sevilla.

Con valores próximos al 2 por 100 se hallan La Coruña, Santander, Alava y Gerona, que amplían y complementan las dos primeras zonas configuradas.

La zona de valores mínimos, inferiores al 1 por 100 de la población activa industrial, es más amplia de la descrita al referirnos a los establecimientos, y comprende a Lugo y Orense; Avila, Palencia, Salamanca, Segovia, Soria y Zamora, en el Valle del Duero; Albacete, Ciudad Real, Cuenca y Guadalajara, Badajoz y Cáceres, en la Sub- 
meseta Sur; Lérida; Huesca y Teruel; Almería, Granada y Huelva, y las dos Provincias Canarias.

Por Autonomias ocupa también el primer lugar Cataluña, con más de la cuarta parte de la población activa industrial. Le siguen, en orden de importancia, el País Valenciano, el País Vasco y Madrid, con más de la décima parte, cada una, del total de personas ocupadas. Entre estas cuatro Autonomías absorben el 60 por 100 del total de población ocupada en la industria. A continuación figuran Andalucía, Castilla-León, Galicia y Asturias, Aragón y Castilla-La Mancha, con valores superiores al 3 por 100 , seguidas de otras cinco con valores inferiores, entre las que figuran, con alrededor del 1 por 100, Rioja, Extremadura, Baleares y Canarias.

Análogamente a lo que ocurre con los establecimientos, si sumamos la población de Madrid y de Castilla-La Mancha, se constituye una Región con el 14,5 de la población activa industrial, que la sitúa en segundo lugar, detrás inmediatamente de Cataluña.

Como se observa, quedan mejor perfiladas las Autonomías y Provincias que destacan por su actividad en el sector industrial.

\section{LA POTENCIA INSTALADA}

La potencia instalada en los establecimientos industriales, en la fecha censal, ascendía a 22.155.980 KW., con un promedio por Provincia de 42.002,46 KW., equivalente al 2 por 100 del total.

Con valores superiores al promedio nacional figuran 15 Provincias, que representan, en conjunto, el 73,3 por 100 de la potencia instalada.

Ocupan los primeros lugares Barcelona y Madrid, seguidas de Asturias, Vizcaya y Guipúzcoa, Valencia, La Coruña, Tarragona y Alava. Y otras seis Provincias con valores entre 2,2 y 2,8.

En conjunto, estas 15 Provincias configuran unas zonas similares, en líneas generales, a las establecidas respecto a las personas ocupadas:

a) Noroeste-Norte: La Coruña, Asturias, Santander, Vizcaya, Guipúzcoa, Alava, Navarra y Zaragoza.

b) Oriental mediterránea: Barcelona, Tarragona, Valencia y Murcia.

c) Madrid y Valladolid.

d) Huelva.

Ofrecen valores próximos al 2 por 100 Pontevedra y Sevilla. 
La zona de valores mínimos, inferiores al 1 por 100, es muy similar a la descrita con referencia a las personas ocupadas, si bien Toledo sustituye a Ciudad Real; no figura Huesca; en Andalucía solamente figuran Almería y Jaén, y a ellas hay que añadir Baleares, Castellón y Rioja.

Por Autonomías ocupan los dos primeros lugares Cataluña y el País Vasco, con cerca de la cuarta parte de la población activa industrial cada una; seguidas de Andalucía y Madrid, con la décima parte cada una; Asturias y el País Valenciano, con el 8 y el 7 por 100. A continuación figuran Castilla-León, Galicia, Aragón y Castilla-La Mancha, con valores superiores al 3 por 100. Con valores mínimos, inferiores al 1 por 100, están Extremadura, Rioja, Baleares y Canarias.

Si, una vez más, consideramos unidas a Madrid y Castilla-La Mancha, la potencia instalada de esta Región se sitúa en el 12,5 por 100, detrás de Cataluña y el País Vasco. Entre estas tres Autonomías disponen de casi el 50 por 100 de la potencia instalada.

\section{Personas ocupadas y potencia instalada POR ESTablecimiento}

Los valores relativos: Personas ocupadas/establecimiento y Potencia instalada/establecimiento, pueden resultar más significativos al objeto de determinar el distinto peso relativo de cada Autonomía y de cada Provincia en orden a su estructura y actividad industrial.

El promedio nacional de personas ocupadas por establecimiento es de 14,4. Tan sólo es superado por 13 Provincias. Entre ellas ocupan los primeros lugares las tres Provincias Vascas, que tienen de 31 a 34 personas ocupadas por establecimiento. A éstas les siguen Madrid, Barcelona, Asturias, Santander y Valladolid, con de 20,5 a 24,1 personas ocupadas por establecimiento; y Navarra, Cádiz, Valencia y Zaragoza, que disponen de 14,6 a 17,6 personas ocupadas por establecimiento industrial.

Las citadas Provincias vienen a configurar las mismas cuatro zonas, ya descritas en orden a las personas ocupadas y potencia instalada, con escasas diferencias:

a) Noroeste-Norte: Asturias, Santander, Vizcaya, Guipúzcoa, Alava, Navarra y Zaragoza.

b) Oriental mediterránea: Barcelona, Gerona y Valencia.

c) Madrid y Valladolid.

d) Cádiz. 
De esta forma se ratifican, una vez más, las cuatro zonas de claro predominio industrial en España.

Con valores entre $10 \mathrm{y}$ el promedio nacional de 14,4 personas ocupadas por establecimiento se hallan las Provincias de Pontevedra, Rioja, Tarragona, Castellón, Alicante y Murcia, Burgos y Guadalajara; y Sevilla, Huelva, Málaga y Jaén, con un cierto grado intermedio de industrialización. Las restantes Provincias presentan los grados inferiores de desarrollo industrial.

Por Autonomias destacan, consecuentemente, en primer lugar, el País Vasco, y a continuación Madrid, Santander y Asturias, Cataluña, Navarra y Valencia, con 14,6 a 32,7 personas ocupadas por establecimiento. Las demás Autonomías oscilan entre 5 y 12 personas ocupadas por industria, correspondiendo los valores mínimos a Extremadura y Castilla-La Mancha, es decir, a la Submeseta Sur, abstracción hecha de la Provincia de Madrid.

El promedio nacional de potencia instalada por establecimiento es de 113,5 KW. Tan sólo es superado por 15 Provincias. También ocupan los primeros lugares las tres Provincias Vascongadas, con de 383,7 a $442,6 \mathrm{KW}$. por establecimiento. Al mismo nivel se halla Asturias. Con más de $200 \mathrm{KW}$. por industria están Huelva, Santander y Valladolid. Otras ocho Provincias disponen de 13,7 a 178,3 KW. por establecimiento.

Estas 15 Provincias configuran, en líneas generales, las mismas zonas ya descritas, aunque con ligeras diferencias:

a) Noroeste-Norte: La Coruña, Asturias, Santander, Vizcaya, Guipúzcoa, Alava, Navarra y Huesca.

b) Oriental mediterránea: Tarragona y Murcia.

c) Meseta: León, Valladolid y Madrid.

d) Cádiz y Huelva.

Debemos consignar que las Provincias de Barcelona y Burgos se aproximan al valor promedio nacional en cuanto a la potencia instalada por establecimiento. En las restantes Provincias, la potencia instalada ofrece valores sensiblemente inferiores al promedio nacional.

Por Autonomias ocupa también el primer lugar el País Vasco y Asturias, a mucha distancia de las demás. Les siguen en orden de importancia Santander, Navarra y Madrid, que disponen de $252 \mathrm{KW}$. la primera citada y 169 y 156 las dos siguientes. Por encima de $100 \mathrm{KW}$. por establecimiento se hallan Murcia, ambas Cas- 
tillas y Aragón. Las demás no llegan a dicho límite y los valores mínimos corresponden a Baleares y Extremadura.

Parece resultar más significativo, al objeto de ordenar adecuadamente las Autonomías y las Provincias, el valor relativo Personas ocupadas/establecimiento, ya que la potencia instalada puede derivarse de ciertas clases de industrias, cuyos establecimientos son escasos en número y desfiguran el valor del área geográfica correspondiente.

La expresada relación de personas ocupadas por establecimiento nos aproximá a una idea del tamaño medio de los establecimientos; pero disponemos de otra información más clara de la distribución de los establecimientos por tamaño, en función del número de personas ocupadas, que tratamos seguidamente.

\section{EL TAMAÑO DE LOS ESTABLECIMIENTOS INDUSTRIALES}

Según el número de personas ocupadas, los establecimientos industriales, eliminados los «no consta», se distribuyen en esta forma:

\begin{tabular}{|c|c|}
\hline & Porcentajes \\
\hline 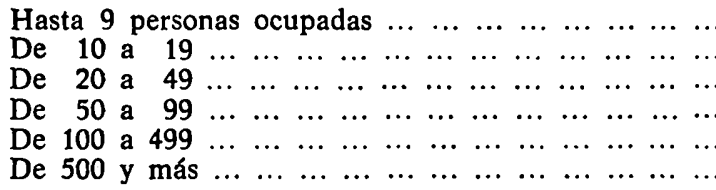 & $\begin{array}{r}76,7 \\
10,1 \\
8,2 \\
2,5 \\
2,2 \\
0,3\end{array}$ \\
\hline
\end{tabular}

En la tabla 2 del Apéndice Estadístico se presenta este detalle por Autonomías y Provincias.

Reduciendo dicha tabla, en lo que a las Autonomias se refiere hemos elaborado la siguiente:

\begin{tabular}{|c|c|c|c|}
\hline & Hasta 19 & De 20 a 99 & De 100 y más \\
\hline 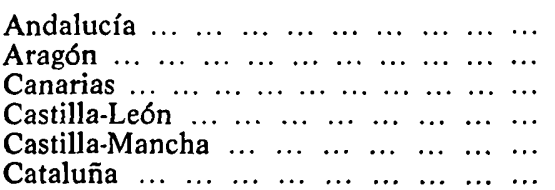 & $\begin{array}{l}91,0 \\
88,4 \\
93,1 \\
92,6 \\
92,9 \\
82,7\end{array}$ & $\begin{array}{r}7,4 \\
9,5 \\
5,5 \\
5,9 \\
6,2 \\
13,7\end{array}$ & $\begin{array}{l}1,6 \\
2,1 \\
1,4 \\
1,5 \\
0,9 \\
3,6\end{array}$ \\
\hline
\end{tabular}




\begin{tabular}{|c|c|c|c|}
\hline & Hasta 19 & De 20 a 99 & De 100 y más \\
\hline 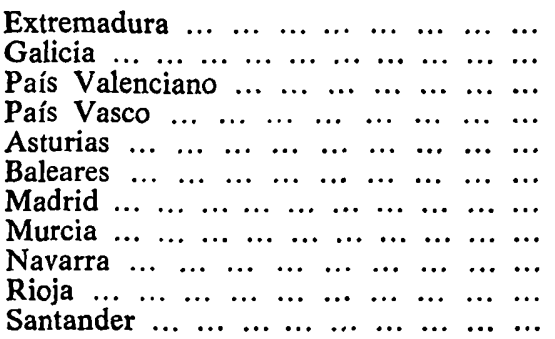 & $\begin{array}{l}95,7 \\
95,6 \\
83,3 \\
75,7 \\
87,5 \\
92,4 \\
81,0 \\
88,3 \\
81,5 \\
84,3 \\
85,2\end{array}$ & $\begin{array}{r}3,5 \\
7,4 \\
14,7 \\
18,0 \\
9,3 \\
6,8 \\
15,3 \\
9,4 \\
14,7 \\
13,8 \\
11,3\end{array}$ & $\begin{array}{l}0,8 \\
1,5 \\
2,0 \\
6,3 \\
3,2 \\
0,8 \\
3,7 \\
2,3 \\
3,8 \\
1,9 \\
3,5\end{array}$ \\
\hline
\end{tabular}

Con establecimientos de más de 100 personas ocupadas sobresalen cuatro Autonomías: el País Vasco y Navarra, Madrid y Cataluña, con tasas superiores al promedio nacional, aunque se acercan a este promedio Santander y Asturias. Configuran tres zonas: una al Norte, con cuatro Autonomías; en el centro, Madrid, y al Este, Cataluña.

Los establecimientos que ocupan de 20 a 99 personas se ubican en un número mayor de Autonomías. Ocupa igualmente el primer lugar el País Vasco, seguido de Madrid, Navarra y el País Valenciano, La Rioja, Cataluña y, finalmente, Santander. Se confirman, una vez más, las tres zonas geográficas reseñadas en el párrafo anterior.

Los establecimientos pequeños, con menos de 20 personas ocupadas, predominan, por el contrario, en Galicia, ambas Castillas -incluidas tres Autonomías-, Andalucía, Aragón, Baleares y Murcia, y las Islas Canarias, con tasas superiores a la nacional, y se le acercan Asturias y Santander. Constituyen una amplísima zona, sin solución de continuidad, que comprende la Meseta - menos Madrid- y los dos grandes valles del Ebro y del Guadalquivir, aparte de las Islas.

Existe, pues, evidentemente, una localización de las Autonomías con mayor desarrollo industrial en el Norte de España, con el País Vasco, Asturias y Santander, Navarra y La Rioja; al Este, Cataluña y el País Valenciano, y en el centro, Madrid, que disponen de establecimientos de mayor capacidad y empleo de mano de obra e incluso de los establecimientos de nivel medio; mientras que los pequeños establecimientos predominan en el resto de las Autonomías, sobre gran parte del área geográfica española. 
Si consideramos, por un momento, los establecimientos por Provincias, observaremos que con establecimientos grandes destacan las tres Provincias Vascas, Navarra, Madrid, Barcelona y Gerona, Asturias y Santander, a las que hay que añadir Sevilla y Burgos, con tasas superiores al promedio nacional. Con valor igual a dicho promedio se sitúa Zaragoza, y se acercan a este promedio Pontevedra, Guadalajara, Castellón, Valencia, Murcia y Cádiz.

Con establecimientos de valor intermedio, en cuanto al número de personas ocupadas, destacan también las tres Provincias Vascas, Santander, Madrid, Navarra, La Rioja, Alicante, Castellon y Valencia, Barcelona y Gerona; y además hay que añadir a Pontevedra, Zaragoza y Cádiz y Ceuta.

Los establecimientos pequeños predominan en todas las Provincias gallegas, Asturias, ambas Castillas, Extremadura y Aragón, Lérida y Tarragona, Andalucía y Murcia, y de las Islas Baleares y Canarias, además de Ceuta y Melilla.

\section{LA UBICACION PROVINCIAL DE LAS PRINCIPALES INDUSTRIAS, SEGUN RAMAS DE LA ACTIVIDAD INDUSTRIAL}

En el Resumen Nacional de la información del Censo Industrial que comentamos, se presenta una información sobre establecimientos, personas ocupadas y potencia instalada, por Provincias, referentes a las 26 ramas de actividad industrial consideradas.

Ello nos permite determinar que el mayor número de establecimientos corresponden a las industrias de productos alimenticios, bebidas y tabaco - 27,2 por 100 del total-; de la madera, corcho y muebles de madera -18,6-; fabricación de productos metálicos, excepto maquinaria y material de transporte -14,1-; captación, depuración y distribución de agua -7,5-; industrias del calzado, vestido y otras confecciones textiles $-6,11-$, e industrias de productos minerales no metálicos - 5,7-.

En orden a las personas ocupadas, destacan también, en primer lugar, las industrias de productos alimenticios, bebidas y tabaco -14,1 por 100 del total-; la fabricación de productos metálicos, excepto maquinaria y material de transporte -11,3-; las industrias de la madera, corcho y muebles de madera -7,9-; las indus- 
trias textil y de calzado, vestido y otras confecciones $-7,1$ cada una-; industrias de productos minerales no metálicos -6,9-; construcción de vehículos automóviles y sus piezas de repuesto -5,7-; construcción de maquinaria y equipo mecánico - 5,4-; y las industrias químicas y del papel y fabricación de artículos de papel, artes gráficas y edición -5 cada una-.

Las actividades industriales que disponen de mayor potencia instalada son: la de producción y primera transformación de metales -21,3 por 100 del total-; la de productos minerales no metálicos -12,2-; la química -10,7-; de productos alimenticios, bebidas y tabaco -9,2-; fabricación de productos metálicos, excepto maquinaria y material de transporte $-8,6-$, y construcción de vehículos automóviles $-5,3$ -

Las industrias con mayor número de personas ocupadas por establecimiento son: las de extracción de petróleo y gas natural, refino de petróleo y extracción y transformación de minerales radioactivos -489-; de extracción, preparación y aglomeración de combustibles sólidos y coquerías -265-; producción y primera transformación de metales -251-; extracción y preparación de minerales metálicos -140-; construcción de vehículos automóviles y sus piezas de repuesto -125-; construcción de máquinas de oficina, incluida su instalación -118-, y fabricación de material electrónico -104-.

Las mayores capacidades de potencia instalada son las correspondientes a las siguientes industrias: extracción de petróleo y gas natural, refino de petróleo y extracción y transformación de minerales radioactivos $-21.817 \mathrm{KW}$. por establecimiento-; producción y primera transformación de metales -8.569-; extracción y preparación de minerales metálicos -2.249-; extracción, preparación y aglomeración de combustibles sólidos y coquerías -1.780-; construcción de vehículos automóviles -922-; química -757-; construcción naval -490-; producción, transporte y distribución de gas - 401-; construcción de maquinaria de oficina -272-; construcción de otro material de transporte -250-; productos minerales no metálicos - 244 , y construcción de maquinaria y material eléctrico -218-.

Expuestos los valores anteriores, dada su importante significación en el conjunto de la industria nacional, parece oportuno hacer una breve referencia a las Provincias que destacan en cada una de las ramas de actividad industrial consideradas. 
La extracción, preparación y aglomeración de combustibles sólidos y coquerías se da principalmente en Oviedo, León y Teruel, en las que se hallan el 80 por 100 de los establecimientos de esta rama, con el 90 por 100 de las personas ocupadas en ella y el 80 por 100 de la potencia instalada.

La extracción de petróleo y gas natural, refino de petróleo y extracción y transformación de materiales radioactivos destaca en Ciudad Real, Santa Cruz de Tenerife, Cádiz, Murcia y Tarragona, con el 14 por 100 de los establecimientos, el 75 de las personas ocupadas y el 71 de la potencia instalada.

En la producción, distribución y transporte de gas destacan Barcelona y Madrid.

En orden a la captación, depuración y distribución de agua, destacan Galicia y Asturias, por el número de establecimientos; Barcelona, Madrid, Valencia y Sevilla, por el número de personas ocupadas, y La Coruña, Las Palmas y Valencia, por la potencia instalada.

La extracción y preparación de minerales metálicos se da básicamente en León, Huelva, Santander y Ciudad Real, con el 66 por 100 de las personas ocupadas y el 43 por 100 de la potencia instalada.

La producción y primera transformación de los metales corresponde esencialmente a Oviedo, Vizcaya, Guipúzcoa, Alava, Barcelona, Santander, Valencia, Madrid y Navarra, con el 75 por 100 de establecimientos, el 85 por 100 de personas ocupadas y el 81 por 100 de la potencia instalada.

La extracción de minerales no metálicos ni energéticos corresponde principalmente a Barcelona, Navarra, Alicante, La Coruña, Huelva, Orense, Oviedo y Santander, con el 25 por 100 de establecimientos, el 50 por 100 de personas ocupadas y el 52 por 100 de potencia instalada.

Las industrias de productos minerales no metálicos destacan en Barcelona, Madrid, Valencia, Castellón, Alicante, Pontevedra, Sevilla y Toledo, con el 42 por 100 de establecimientos, el 57 por 100 de personas ocupadas y el 60 por 100 de potencia instalada.

En la industria química destacan, a su vez, Barcelona, Madrid, Vizcaya, Santander, Tarragona y Valencia, Gerona y Zaragoza, con el 59 por 100 de establecimientos, el 73 por 100 de personas ocupadas y el 49 por 100 de potencia instalada.

La fabricación de productos metálicos -excepto maquinaria y 
material de transporte- se da principalmente en Barcelona, Madrid, Vizcaya, Guipúzcoa, Valencia, Zaragoza, Alava y Oviedo, con el 49 por 100 de establecimientos, el 70 por 100 de personas ocupadas y el 75 por 100 de potencia instalada.

En la construcción de maquinaria y equipo mecánico sobresalen Barcelona, Madrid, Guipúzcoa, Vizcaya, Zaragoza y Valencia, con el 49 por 100 de establecimientos, el 71 por 100 de personas ocupadas y el 67 por 100 de potencia instalada.

La construcción de maquinaria de oficina corresponde esencialmente a Barcelona, Madrid, Toledo y Guipúzcoa, con el 61 por 100 de establecimientos, el 96 por 100 de personas ocupadas y el 99 por 100 de potencia instalada.

En la construcción de maquinaria y material eléctrico sobresalen Barcelona, Madrid, Vizcaya, Guipúzcoa y Zaragoza, con el 60 por 100 de establecimientos, el 74 por 100 de personas ocupadas y el 70 por 100 de potencia instalada.

La fabricación de material electrónico corresponde a Madrid, Barcelona, Navarra, Guipúzcoa y Málaga, con el 63 por 100 de establecimientos, el 90 por 100 de personas ocupadas y el 83 por 100 de potencia instalada.

En la construcción de vehículos automóviles destacan Barcelona, Madrid, Valladolid, Valencia, Pontevedra y Navarra, con el 50 por 100 de establecimientos, el 77 por 100 de personas ocupadas y el 79 por 100 de potencia instalada.

En la construcción naval sobresalen Vizcaya, Cádiz, La Coruña, Murcia, Pontevedra, Oviedo y Valencia, con el 51 por 100 de establecimientos, el 81 por 100 de personas ocupadas y el 91 por 100 de potencia instalada.

En la construcción de otro material de transporte destacan Barcelona, Madrid, Valencia, Sevilla, Valladolid, Guipúzcoa, Zaragoza, Vizcaya, Alava y León, con el 43 por 100 de establecimientos, el 85 por 100 de personas ocupadas y el 88 por 100 de potencia instalada.

La fabricación de instrumentos de precisión y óptica se da principalmente en Madrid, Barcelona, Guipúzcoa, Oviedo, Vizcaya y Valencia, con el 67 por 100 de establecimientos, el 90 por 100 de personas ocupadas y el 92 por 100 de potencia instalada.

En las industrias de productos alimenticios, bebidas y tabaco, sobresalen Barcelona, Madrid, Valencia, Sevilla, Murcia, Cádiz, Pontevedra, Navarra, Alicante, La Coruña y Jaén, con el 35 por 100 de 
establecimientos, el 49 por 100 de personas ocupadas y el 41 por 100 de potencia instalada.

En la industria textil destacan Barcelona, Gerona, Valencia y Alicante, con el 76 por 100 de establecimientos, el 78 por 100 de personas ocupadas y el 74 por 100 de potencia instalada.

La industria del cuero se da básicamente en Barcelona, Valencia, Madrid, Alicante y Cádiz, con el 59 por 100 de establecimientos, el 69 por 100 de personas ocupadas y el 65 por 100 de potencia instalada.

En la industria del calzado y vestido ocupan los primeros lugares Alicante, Barcelona, Madrid, Valencia, Zaragoza, Baleares y Albacete, con el 50 por 100 de establecimientos, el 65 por 100 de personas ocupadas y el 63 por 100 de potencia instalada.

En la industria de la madera, corcho y muebles de madera sobresalen Valencia, Barcelona, Madrid, Alicante, La Coruña, Guipúzcoa, Murcia y Gerona, con el 42 por 100 de establecimientos, el 52 por 100 de personas ocupadas y el 47 por 100 de potencia instalada.

En la industria del papel y fabricación de artículos de papel destacan Barcelona, Madrid, Guipúzcoa, Vizcaya, Navarra, Zaragoza, Gerona y Alicante, con el 66 por 100 de establecimientos, el 73 por 100 de personas ocupadas y el 69 por 100 de potencia instalada.

En las industrias de transformación del caucho y materias plásticas sobresalen Barcelona, Vizcaya, Madrid, Alicante, Guipúzcoa, Valencia y Alava, con el 67 por 100 de establecimientos, el 73 por 100 de personas ocupadas, e igual porcentaje de potencia instalada.

$\mathrm{Y}$, finalmente, en otras industrias manufactureras, destacan también Barcelona, Alicante, Madrid, Valencia, Baleares, Córdoba y Alava, con el 67 por 100 de establecimientos, el 78 por 100 de personas ocupadas y el 65 por 100 de potencia instalada.

De los párrafos anteriores, en que se ha expuesto para cada rama de actividad industrial las Provincias que destacan, por orden de importancia en cada una de ellas, indicando el porcentaje de establecimientos, personas ocupadas y potencia instalada que representan las Provincias enumeradas dentro de la respectiva rama de actividad industrial, se deduce la fuerte concentración de la industria española, como ya se indicó en el apartado II. 
REVL-1981, núm. 210. BALLESTER ROS, IGNACIO. ALGUNAS CARACTERISTICAS DE LOS ESTA... 336

REVISTA DE ESTUDIOS DE LA VIDA LOCAL

\section{APENDICE ESTADISTICO}

1. NÚmero DE ESTABlecimientos, PERSONAS OCUPADAS Y POTENCIA INSTALADA

CENSO INDUSTRIAL DE ESPANAA. ANTO 1978

A) Cifras absolutas

\begin{tabular}{|c|c|c|c|}
\hline REGIONES Y PROVINCIAS & $\begin{array}{c}\text { Número de } \\
\text { establecimientos }\end{array}$ & $\begin{array}{l}\text { Personas } \\
\text { ocupadas }\end{array}$ & $\begin{array}{l}\text { Potencia } \\
\text { instalada } \\
\text { K. } \mathbf{w} .\end{array}$ \\
\hline $\begin{array}{lllllllll}\text { ANDALUCIA } & \ldots & \ldots & \ldots & \ldots & \ldots & \ldots & \ldots\end{array}$ & 22.948 & 232.484 & 2.100 .123 \\
\hline 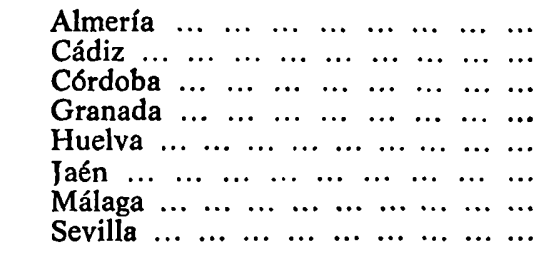 & $\begin{array}{l}2.171 \\
2.464 \\
3.171 \\
3.262 \\
1.751 \\
2.977 \\
2.540 \\
4.612\end{array}$ & $\begin{array}{r}8.985 \\
39.061 \\
27.362 \\
18.237 \\
20.429 \\
30.218 \\
27.271 \\
60.921\end{array}$ & $\begin{array}{r}64.464 \\
300.315 \\
210.795 \\
225.119 \\
487.009 \\
187.397 \\
239.056 \\
385.986\end{array}$ \\
\hline 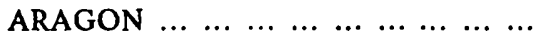 & 8.841 & 104.721 & 935.165 \\
\hline $\begin{array}{llllllllll}\text { Huesca } & \ldots & \ldots & \ldots & \ldots & \ldots & \ldots & \ldots & \ldots & \ldots \\
\text { Teruel } & \ldots & \ldots & \ldots & \ldots & \ldots & \ldots & \ldots & \ldots & \ldots \\
\text { Zaragoza } & \ldots & \ldots & \ldots & \ldots & \ldots & \ldots & \ldots & \ldots\end{array}$ & $\begin{array}{l}2.093 \\
1.456 \\
5.292\end{array}$ & $\begin{array}{l}15.043 \\
12.394 \\
77.284\end{array}$ & $\begin{array}{l}317.345 \\
118.240 \\
499.580\end{array}$ \\
\hline $\begin{array}{lllllllll}\text { CANARIAS } & \ldots & \ldots & \ldots & \ldots & \ldots & \ldots & \ldots & \ldots\end{array}$ & 3.572 & 31.084 & 166.480 \\
\hline $\begin{array}{llllllll}\text { Las Palmas } & \ldots & \ldots & \ldots & \ldots & \ldots & \ldots & \ldots \\
\text { Santa Cruz de } & \text { Tenerife } & \ldots & \ldots & \ldots & \ldots\end{array}$ & $\begin{array}{l}1.572 \\
2.000\end{array}$ & $\begin{array}{l}15.427 \\
15.657\end{array}$ & $\begin{array}{l}70.020 \\
96.460\end{array}$ \\
\hline $\begin{array}{llllllll}\text { CASTILLA-LEON } & \ldots & \ldots & \ldots & \ldots & \ldots & \ldots\end{array}$ & 17.669 & 163.460 & 1.370 .927 \\
\hline $\begin{array}{llllllllll}\text { Avila } & \ldots & \ldots & \ldots & \ldots & \ldots & \ldots & \ldots & \ldots & \ldots \\
\text { Burgos } & \ldots & \ldots & \ldots & \ldots & \ldots & \ldots & \ldots & \ldots & \ldots \\
\text { León } & \ldots & \ldots & \ldots & \ldots & \ldots & \ldots & \ldots & \ldots & \ldots \\
\text { Palencia } & \ldots & \ldots & \ldots & \ldots & \ldots & \ldots & \ldots & \ldots \\
\text { Salamanca } & \ldots & \ldots & \ldots & \ldots & \ldots & \ldots & \ldots & \ldots \\
\text { Segovia } & \ldots & \ldots & \ldots & \ldots & \ldots & \ldots & \ldots & \ldots & \ldots \\
\text { Segovia } & \ldots & \ldots & \ldots & \ldots & \ldots & \ldots & \ldots & \ldots \\
\text { Soria } & \ldots & \ldots & \ldots & \ldots & \ldots & \ldots & \ldots & \ldots \\
\text { Valladolid } & \ldots & \ldots & \ldots & \ldots & \ldots & \ldots & \ldots & \ldots & \ldots \\
\text { Zamora } & \ldots & \ldots & \ldots & \ldots & \ldots & \ldots & \ldots & \ldots\end{array}$ & $\begin{array}{l}1.285 \\
2.546 \\
3.695 \\
1.221 \\
2.481 \\
1.434 \\
1.021 \\
2.254 \\
1.732\end{array}$ & $\begin{array}{r}5.629 \\
31.485 \\
34.314 \\
12.101 \\
14.239 \\
8.000 \\
6.099 \\
46.212 \\
5.381\end{array}$ & $\begin{array}{r}22.981 \\
266.656 \\
232.711 \\
151.504 \\
61.360 \\
49.948 \\
38.842 \\
497.163 \\
49.762\end{array}$ \\
\hline $\begin{array}{llllll}\text { CASTILLA-MANCHA } & \ldots & \ldots & \ldots & \ldots & \ldots\end{array}$ & 13.347 & 89.757 & 703.124 \\
\hline 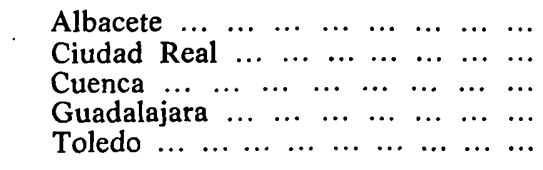 & $\begin{array}{l}2.580 \\
3.853 \\
1.847 \\
1.127 \\
3.940\end{array}$ & $\begin{array}{r}19.421 \\
20.912 \\
7.811 \\
11.780 \\
29.833\end{array}$ & $\begin{array}{r}64.696 \\
270.555 \\
67.919 \\
93.987 \\
205.967\end{array}$ \\
\hline $\begin{array}{llllllllll}\text { CATALUÑA } & \ldots & \ldots & \ldots & \ldots & \ldots & \ldots & \ldots & \ldots\end{array}$ & 37.364 & 716.196 & 3.989 .462 \\
\hline $\begin{array}{llllllllll}\text { Barcelona } & \ldots & \ldots & \ldots & \ldots & \ldots & \ldots & \ldots & \ldots \\
\text { Gerona } & \ldots & \ldots & \ldots & \ldots & \ldots & \ldots & \ldots & \ldots\end{array}$ & $\begin{array}{r}26.429 \\
3.431\end{array}$ & $\begin{array}{r}597.480 \\
53.064\end{array}$ & $\begin{array}{r}2.810 .419 \\
304.681\end{array}$ \\
\hline
\end{tabular}




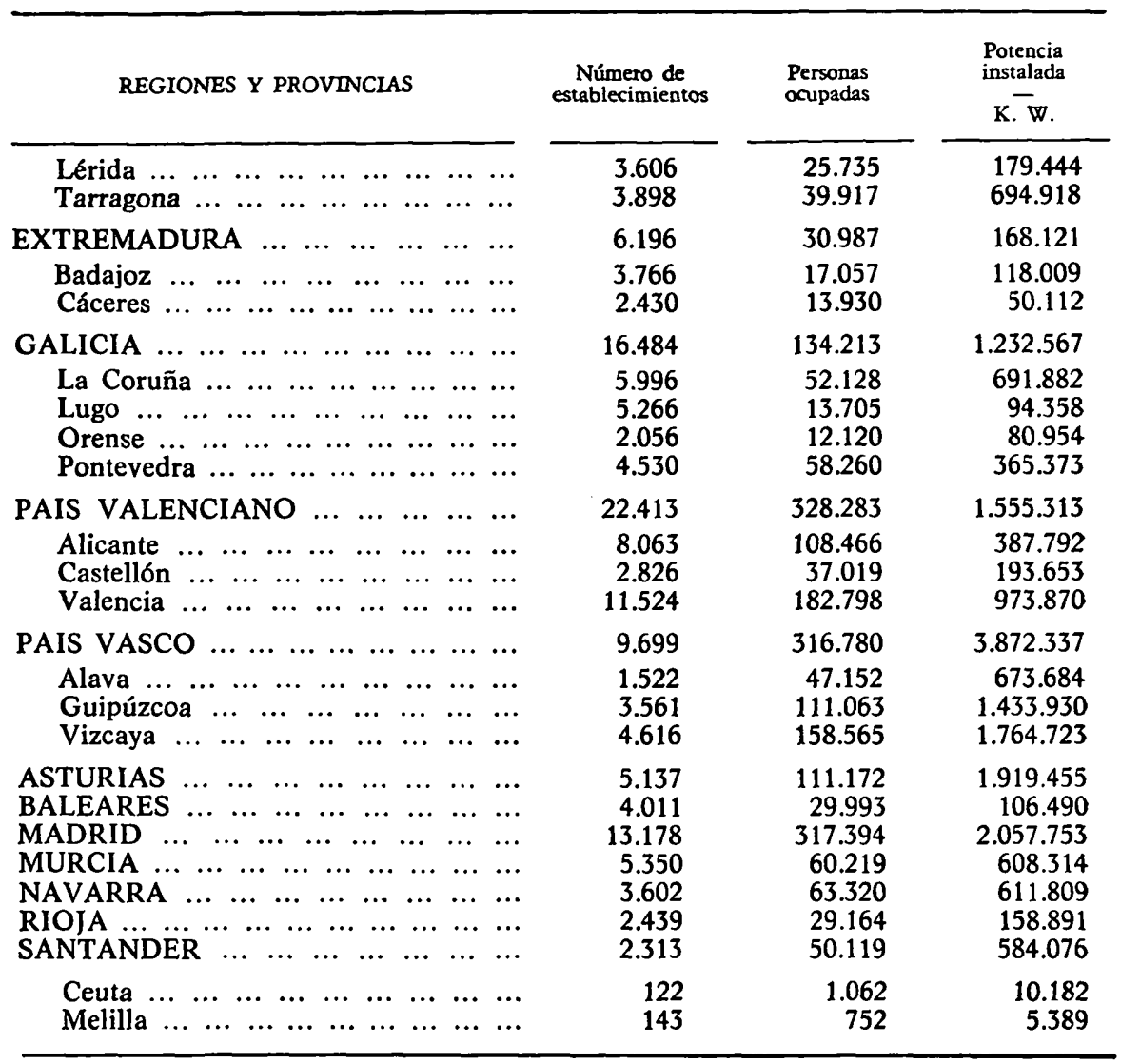

\section{B) Porcentajes}

\begin{tabular}{|c|c|c|c|}
\hline REGIONES Y PROVINCIAS & $\begin{array}{c}\text { Número de } \\
\text { establecimientos }\end{array}$ & $\begin{array}{l}\text { Personas } \\
\text { ocupadas }\end{array}$ & $\begin{array}{l}\text { Potencia } \\
\text { instalada } \\
\text { K. } \mathrm{w} .\end{array}$ \\
\hline $\begin{array}{llllllll}\text { ANDALUCIA } & \ldots & \ldots & \ldots & \ldots & \ldots & \ldots & \ldots\end{array}$ & 11,9 & 8,3 & 9,5 \\
\hline 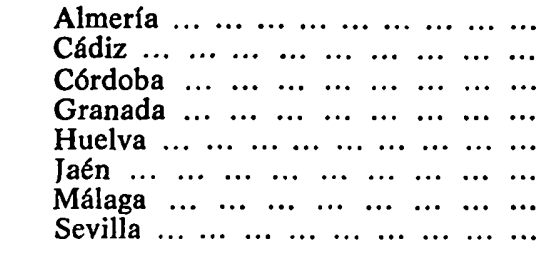 & $\begin{array}{l}1,1 \\
1,3 \\
1,6 \\
1,8 \\
0,9 \\
1,5 \\
1,3 \\
2,4\end{array}$ & $\begin{array}{l}0,3 \\
1,4 \\
1,0 \\
0,6 \\
0,7 \\
1,1 \\
1,0 \\
2,2\end{array}$ & $\begin{array}{l}0,3 \\
1,4 \\
1,0 \\
1,0 \\
2,2 \\
0,8 \\
1,1 \\
1,7\end{array}$ \\
\hline ARAGON $\ldots \ldots \ldots \ldots \ldots$ & 4,5 & 3,6 & 4,2 \\
\hline 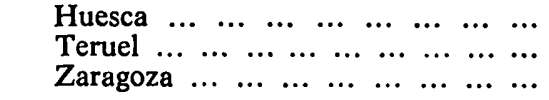 & $\begin{array}{l}1,1 \\
0,7 \\
2,7\end{array}$ & $\begin{array}{l}0,5 \\
0,4 \\
2,7\end{array}$ & $\begin{array}{l}1,4 \\
0,5 \\
2,3\end{array}$ \\
\hline
\end{tabular}




\begin{tabular}{|c|c|c|c|}
\hline REGIONES Y PROVINCIAS & $\begin{array}{c}\text { Número de } \\
\text { establecimientos }\end{array}$ & $\begin{array}{l}\text { Personas } \\
\text { ocupadas }\end{array}$ & $\begin{array}{l}\text { Potencia } \\
\text { instalada } \\
\text { K. W. }\end{array}$ \\
\hline $\begin{array}{rllllllll}\text { CANARIAS } & \ldots & \ldots & \ldots & \ldots & \ldots & \ldots & \ldots & \ldots \\
\text { Las Palmas } & \ldots & \ldots & \ldots & \ldots & \ldots & \ldots & \ldots \\
\text { Santa Cruz } & \text { de } & \text { Tenerife } & \ldots & \ldots & \ldots\end{array}$ & $\begin{array}{l}1,8 \\
0,8 \\
1,0\end{array}$ & $\begin{array}{l}1,1 \\
0,5 \\
0,6\end{array}$ & $\begin{array}{l}0,7 \\
0,3 \\
0,4\end{array}$ \\
\hline 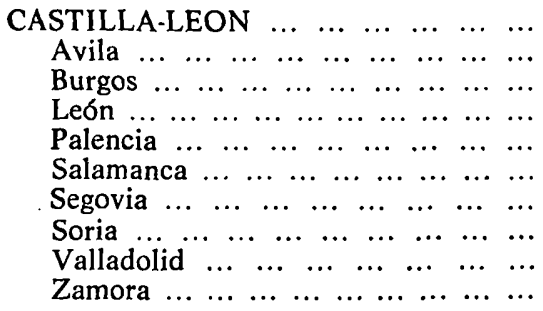 & $\begin{array}{l}9,1 \\
0,7 \\
1,3 \\
1,9 \\
0,6 \\
1,3 \\
0,7 \\
0,5 \\
1,2 \\
0,9\end{array}$ & $\begin{array}{l}5,8 \\
0,2 \\
1,1 \\
1,2 \\
0,4 \\
0,5 \\
0,3 \\
0,3 \\
1,6 \\
0,2\end{array}$ & $\begin{array}{l}6,2 \\
0,1 \\
1,2 \\
1,1 \\
0,7 \\
0,3 \\
0,2 \\
0,2 \\
2,2 \\
0,2\end{array}$ \\
\hline 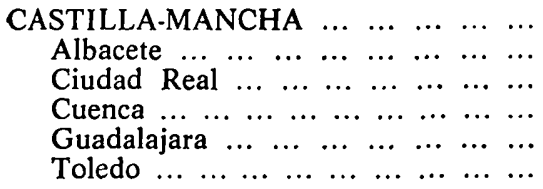 & $\begin{array}{l}6,8 \\
1,3 \\
2,0 \\
0,9 \\
0,6 \\
2,0\end{array}$ & $\begin{array}{l}3,2 \\
0,7 \\
0,7 \\
0,3 \\
0,4 \\
1,1\end{array}$ & $\begin{array}{l}3,2 \\
0,3 \\
1,2 \\
0,4 \\
0,4 \\
0,9\end{array}$ \\
\hline 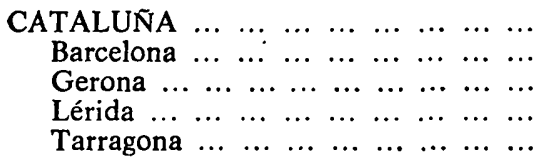 & $\begin{array}{r}19,1 \\
13,5 \\
1,8 \\
1,8 \\
2,0\end{array}$ & $\begin{array}{r}25,5 \\
21,3 \\
1,9 \\
0,9 \\
1,4\end{array}$ & $\begin{array}{r}18,0 \\
12,7 \\
1,4 \\
0,8 \\
3,1\end{array}$ \\
\hline 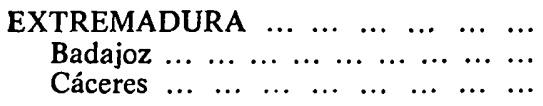 & $\begin{array}{l}3,2 \\
1,9 \\
1,3\end{array}$ & $\begin{array}{l}1,1 \\
0,6 \\
0,5\end{array}$ & $\begin{array}{l}0,8 \\
0,5 \\
0,3\end{array}$ \\
\hline 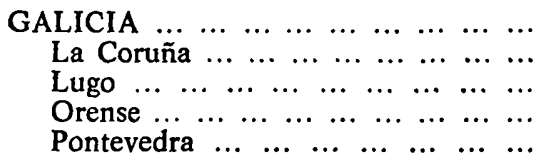 & $\begin{array}{l}8,6 \\
3,1 \\
2,2 \\
1,0 \\
2,3\end{array}$ & $\begin{array}{l}4,8 \\
1,9 \\
0,5 \\
0,4 \\
2,0\end{array}$ & $\begin{array}{l}5,6 \\
3,1 \\
0,5 \\
0,4 \\
1,6\end{array}$ \\
\hline $\begin{array}{rllllllll}\text { PAIS VALENCIANO } & \ldots & \ldots & \ldots & \ldots & \ldots \\
\text { Alicante } & \ldots & \ldots & \ldots & \ldots & \ldots & \ldots & \ldots & \ldots \\
\text { Castellón } & \ldots & \ldots & \ldots & \ldots & \ldots & \ldots & \ldots & \ldots \\
\text { Valencia } & \ldots & \ldots & \ldots & \ldots & \ldots & \ldots & \ldots & \ldots\end{array}$ & $\begin{array}{r}11,5 \\
4,1 \\
1,5 \\
5,9\end{array}$ & $\begin{array}{r}11,7 \\
3,9 \\
1,3 \\
6,5\end{array}$ & $\begin{array}{l}7,0 \\
1,8 \\
0,8 \\
4,4\end{array}$ \\
\hline 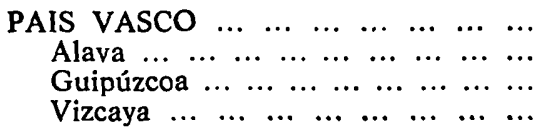 & $\begin{array}{l}5,0 \\
0,8 \\
1,8 \\
2,4\end{array}$ & $\begin{array}{r}11,3 \\
1,7 \\
4,0 \\
5,6\end{array}$ & $\begin{array}{r}17,5 \\
3,0 \\
6,5 \\
8,0\end{array}$ \\
\hline 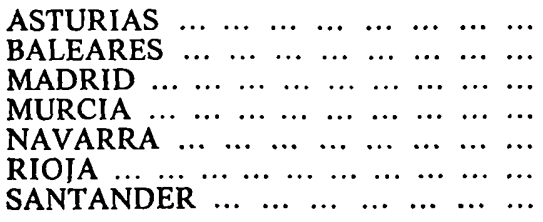 & $\begin{array}{l}2,6 \\
2,1 \\
6,8 \\
2,7 \\
1,8 \\
1,3 \\
1,2\end{array}$ & $\begin{array}{r}4,0 \\
1,1 \\
11,3 \\
2,1 \\
2,3 \\
1,0 \\
1,8\end{array}$ & $\begin{array}{l}8,7 \\
0,5 \\
9,3 \\
2,7 \\
2,8 \\
0,7 \\
2,6\end{array}$ \\
\hline $\begin{array}{cccccccccc}\text { Ceuta } & \ldots & \ldots & \ldots & \ldots & \ldots & \ldots & \ldots & \ldots & \ldots \\
\text { Melilla } & \ldots & \ldots & \ldots & \ldots & \ldots & \ldots & \ldots & \ldots & \ldots\end{array}$ & $\begin{array}{l}0,1 \\
0,1\end{array}$ & - & - \\
\hline
\end{tabular}


C) Personas ocupadas y potencia instalada por establecimiento

\begin{tabular}{|c|c|c|}
\hline KEGIOINES Y PROVINCIAS & $\begin{array}{c}\text { Personas } \\
\text { ocupadas pe: } \\
\text { establecimiento }\end{array}$ & $\begin{array}{c}\text { Potencia } \\
\text { instalada por } \\
\text { establecimiento }\end{array}$ \\
\hline $\begin{array}{llllllll}\text { ANDALUCIA } & \ldots & \ldots & \ldots & \ldots & \ldots & \ldots & \ldots\end{array}$ & 10,1 & 91,5 \\
\hline 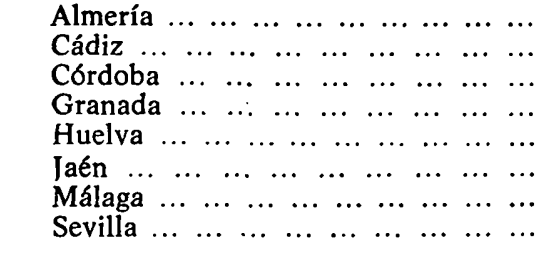 & $\begin{array}{r}4,1 \\
15,9 \\
8,6 \\
5,6 \\
11,7 \\
10,2 \\
10,7 \\
13,2\end{array}$ & $\begin{array}{r}29,7 \\
121,9 \\
66,5 \\
69,0 \\
278,1 \\
62,9 \\
94,1 \\
83,7\end{array}$ \\
\hline $\begin{array}{lllllllllll}\text { ARAGON } & \ldots & \ldots & \ldots & \ldots & \ldots & \ldots & \ldots & \ldots & \ldots\end{array}$ & 11,8 & 105,8 \\
\hline $\begin{array}{lllllllll}\text { Huesca } & \ldots & \ldots & \ldots & \ldots & \ldots & \ldots & \ldots & \ldots \\
\text { Teruel } & \ldots & \ldots & \ldots & \ldots & \ldots & \ldots & \ldots & \ldots \\
\text { Zaragoza } & \ldots & \ldots & \ldots & \ldots & \ldots & \ldots & \ldots & \ldots\end{array}$ & $\begin{array}{r}7,2 \\
8,5 \\
14,6\end{array}$ & $\begin{array}{r}151,6 \\
81,2 \\
94,4\end{array}$ \\
\hline $\begin{array}{rllllllll}\text { CANARIAS } & \ldots & \ldots & \ldots & \ldots & \ldots & \ldots & \ldots & \ldots \\
\text { Las Palmas } & \ldots & \ldots & \ldots & \ldots & \ldots & \ldots & \ldots & \ldots \\
\text { Santa Cruz de } & \text { Tenerife } & \ldots & \ldots & \ldots & \ldots\end{array}$ & $\begin{array}{l}8,7 \\
9,8 \\
7,8\end{array}$ & $\begin{array}{l}46,6 \\
44,5 \\
48,2\end{array}$ \\
\hline 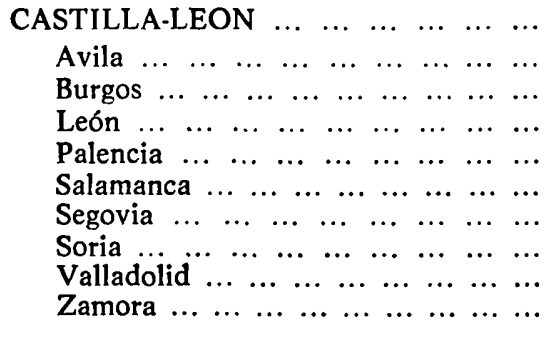 & $\begin{array}{r}9,3 \\
4,4 \\
12,4 \\
9,3 \\
9,9 \\
5,7 \\
5,6 \\
6,0 \\
20,5 \\
3,1\end{array}$ & $\begin{array}{r}77,6 \\
17,9 \\
104,7 \\
63,0 \\
124,1 \\
24,7 \\
34,8 \\
38,0 \\
220,6 \\
28,7\end{array}$ \\
\hline 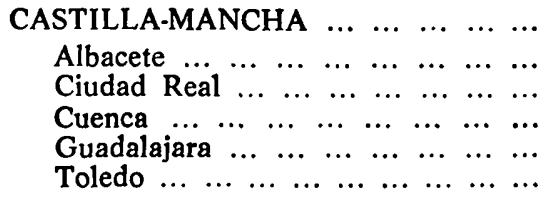 & $\begin{array}{r}6,7 \\
7,5 \\
5,4 \\
4,2 \\
10,5 \\
7,6\end{array}$ & $\begin{array}{r}102,7 \\
25,1 \\
94,8 \\
36,8 \\
83,9 \\
52,3\end{array}$ \\
\hline 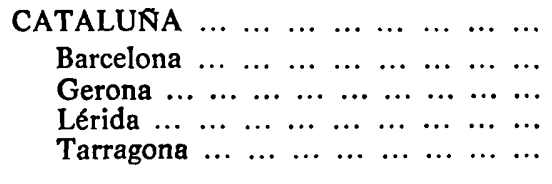 & $\begin{array}{r}19,2 \\
22,6 \\
15,5 \\
7,1 \\
10,2\end{array}$ & $\begin{array}{r}106,8 \\
106,3 \\
88,8 \\
49,8 \\
178,3\end{array}$ \\
\hline 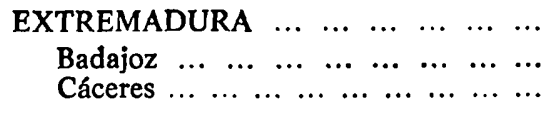 & $\begin{array}{l}5,0 \\
4,5 \\
5,7\end{array}$ & $\begin{array}{l}27,1 \\
31,3 \\
20,6\end{array}$ \\
\hline 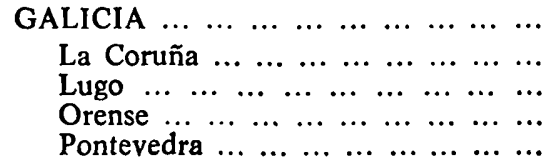 & $\begin{array}{r}8,0 \\
8,7 \\
3,2 \\
5,9 \\
12,4\end{array}$ & $\begin{array}{r}73,2 \\
115,4 \\
22,1 \\
39,4 \\
80,7\end{array}$ \\
\hline
\end{tabular}




\begin{tabular}{|c|c|c|}
\hline REGIONES Y PROVINCIAS & $\begin{array}{c}\text { Personas } \\
\text { ocupadas por } \\
\text { establecimiento }\end{array}$ & $\begin{array}{c}\text { Potencia } \\
\text { instalada por } \\
\text { establecimiento }\end{array}$ \\
\hline PAIS VALENCIANO $\ldots \ldots \ldots c c c c$ & 14,6 & 69,4 \\
\hline $\begin{array}{lllllllll}\text { Alicante } & \ldots & \ldots & \ldots & \ldots & \ldots & \ldots & \ldots & \ldots \\
\text { Castellón } & \ldots & \ldots & \ldots & \ldots & \ldots & \ldots & \ldots & \ldots \\
\text { Valencia } & \ldots & \ldots & \ldots & \ldots & \ldots & \ldots & \ldots & \ldots\end{array}$ & $\begin{array}{l}13,5 \\
13,1 \\
15,9\end{array}$ & $\begin{array}{l}48,1 \\
68,5 \\
84,5\end{array}$ \\
\hline $\begin{array}{llllllllll}\text { PAIS } V A S C O & \ldots & \ldots & \ldots & \ldots & \ldots & \ldots & \ldots\end{array}$ & 32,7 & 399,3 \\
\hline $\begin{array}{llllllllll}\text { Alava } & \ldots & \ldots & \ldots & \ldots & \ldots & \ldots & \ldots & \ldots & \ldots \\
\text { Guipúzcoa } & \ldots & \ldots & \ldots & \ldots & \ldots & \ldots & \ldots & \ldots \\
\text { Vizcaya } & \ldots & \ldots & \ldots & \ldots & \ldots & \ldots & \ldots & \ldots & \ldots\end{array}$ & $\begin{array}{l}31,0 \\
31,2 \\
34,4\end{array}$ & $\begin{array}{l}442,6 \\
402,7 \\
382,3\end{array}$ \\
\hline 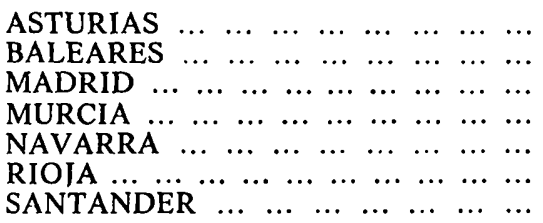 & $\begin{array}{r}21,6 \\
7,5 \\
24,1 \\
11,3 \\
17,6 \\
12,0 \\
21,7\end{array}$ & $\begin{array}{r}373,7 \\
26,5 \\
156,2 \\
113,7 \\
169,9 \\
65,1 \\
252,5\end{array}$ \\
\hline $\begin{array}{llllllllll}\text { Ceuta } & \ldots & \ldots & \ldots & \ldots & \ldots & \ldots & \ldots & \ldots & \ldots \\
\text { Melilla } & \ldots & \ldots & \ldots & \ldots & \ldots & \ldots & \ldots & \ldots & \ldots\end{array}$ & $\begin{array}{l}8,7 \\
5,3\end{array}$ & $\begin{array}{l}83,5 \\
37,7\end{array}$ \\
\hline
\end{tabular}

2. Tamaño de los establecimientos. Porcentajes

\begin{tabular}{|c|c|c|c|c|c|c|}
\hline $\begin{array}{l}\text { REGIONES } \\
\mathrm{Y} \text { PROVINCIAS }\end{array}$ & Hasta 9 & $\begin{array}{l}\text { De } 10 \\
\text { a } 19\end{array}$ & $\begin{array}{c}\text { De } 20 \\
\text { a } 49\end{array}$ & $\begin{array}{l}\text { De } 50 \\
\text { a } 99\end{array}$ & $\begin{array}{l}\text { De } 100 \\
\text { a } 499\end{array}$ & $\begin{array}{l}\text { De } 500 \\
\text { y más }\end{array}$ \\
\hline ANDALUCIA $\ldots \ldots \ldots$ & 82,3 & 8,7 & 5,9 & 1,5 & 1,4 & 0,2 \\
\hline 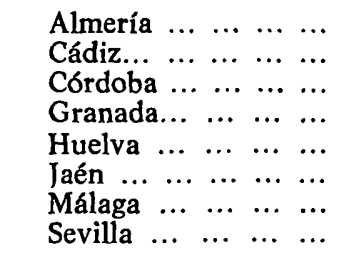 & $\begin{array}{l}91,5 \\
75,9 \\
81,1 \\
88,1 \\
84,9 \\
78,1 \\
84,3 \\
78,5\end{array}$ & $\begin{array}{r}6,0 \\
10,8 \\
9,4 \\
6,2 \\
7,2 \\
10,8 \\
8,3 \\
9,6\end{array}$ & $\begin{array}{l}1,8 \\
8,0 \\
6,5 \\
3,7 \\
5,0 \\
8,2 \\
4,8 \\
7,2\end{array}$ & $\begin{array}{l}0,6 \\
3,0 \\
1,6 \\
0,8 \\
1,2 \\
1,4 \\
1,2 \\
2,0\end{array}$ & $\begin{array}{l}0,1 \\
1,8 \\
1,3 \\
0,8 \\
1,1 \\
1,3 \\
1,2 \\
2,3\end{array}$ & $\begin{array}{l}\overline{0,5} \\
0,1 \\
0,1 \\
0,6 \\
0,2 \\
0,2 \\
0,4\end{array}$ \\
\hline ARAGON $\ldots \ldots \ldots$ & 77,7 & 10,7 & 7,5 & 2,0 & 1,8 & 0,3 \\
\hline $\begin{array}{lllll}\text { Huesca } & \ldots & \ldots & \ldots & \ldots \\
\text { Teruel... } & \ldots & \ldots & \ldots & \ldots \\
\text { Zaragoza... } & \ldots & \ldots & \ldots\end{array}$ & $\begin{array}{l}85,6 \\
88,3 \\
72,3\end{array}$ & $\begin{array}{r}6,7 \\
5,1 \\
13,5\end{array}$ & $\begin{array}{l}5,3 \\
4,2 \\
9,2\end{array}$ & $\begin{array}{l}1,3 \\
0,9 \\
2,5\end{array}$ & $\begin{array}{l}0,9 \\
1,0 \\
2,3\end{array}$ & $\begin{array}{l}0,2 \\
0,5 \\
0,2\end{array}$ \\
\hline CANARIAS... $\ldots \ldots \ldots$ & 86,6 & 6,5 & 4,1 & 1,4 & 1,3 & 0,1 \\
\hline $\begin{array}{l}\text { Las Palmas } \ldots \ldots \ldots \\
\text { S. C. de Tenerife } \ldots\end{array}$ & $\begin{array}{l}83,4 \\
89,1\end{array}$ & $\begin{array}{l}7,4 \\
5,8\end{array}$ & $\begin{array}{l}5,7 \\
2,9\end{array}$ & $\begin{array}{l}1,8 \\
1,0\end{array}$ & $\begin{array}{l}1,7 \\
1,0\end{array}$ & $\overline{0,2}$ \\
\hline CASTILLA-LEON... ... & 86,4 & 6,2 & 4,6 & 1,3 & 1,3 & 0,2 \\
\hline 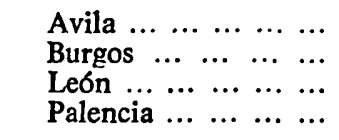 & $\begin{array}{l}92,1 \\
80,7 \\
86,3 \\
86,4\end{array}$ & $\begin{array}{l}4,5 \\
7,4 \\
5,9 \\
5,2\end{array}$ & $\begin{array}{l}2,7 \\
5,8 \\
4,6 \\
4,8\end{array}$ & $\begin{array}{l}0,4 \\
2,9 \\
1,2 \\
1,8\end{array}$ & $\begin{array}{l}0,2 \\
2,8 \\
1,7 \\
1,5\end{array}$ & $\begin{array}{l}0,1 \\
0,4 \\
0,3 \\
0,3\end{array}$ \\
\hline
\end{tabular}


ESTADISTICA

\begin{tabular}{|c|c|c|c|c|c|c|}
\hline $\begin{array}{l}\text { REGIONES } \\
Y \text { PROVINCIAS }\end{array}$ & Hasta 9 & $\begin{array}{c}\mathrm{De} 10 \\
\text { a } 19\end{array}$ & $\begin{array}{c}\mathrm{De} 20 \\
\text { a } 49\end{array}$ & $\begin{array}{c}\text { De } 50 \\
\text { a } 99\end{array}$ & $\begin{array}{l}\text { De } 100 \\
\text { a } 499\end{array}$ & $\begin{array}{l}\text { De } 500 \\
\text { y más }\end{array}$ \\
\hline $\begin{array}{lllll}\text { Salamanca } & \ldots & \ldots & \ldots \\
\text { Segovia } & \ldots & \ldots & \ldots & \ldots \\
\text { Soria } \ldots & \ldots & \ldots & \ldots & \ldots \\
\text { Valladolid } & \ldots & \ldots & \ldots \\
\text { Zamora } & \ldots & \ldots & \ldots & \ldots \\
\end{array}$ & $\begin{array}{l}89,4 \\
87,1 \\
85,8 \\
79,2 \\
93,9\end{array}$ & $\begin{array}{l}5,3 \\
6,8 \\
6,6 \\
9,4 \\
3,7\end{array}$ & $\begin{array}{l}3,5 \\
5,2 \\
5,1 \\
7,3 \\
1,9\end{array}$ & $\begin{array}{l}0,9 \\
0,3 \\
1,1 \\
1,8 \\
0,2\end{array}$ & $\begin{array}{l}0,9 \\
0,4 \\
1,2 \\
1,9 \\
0,3\end{array}$ & $\begin{array}{l}- \\
0,2 \\
0,2 \\
0,4 \\
-\end{array}$ \\
\hline CASTILLA-MANCHA... & 86,2 & 6,7 & 4,7 & 1,5 & 0,8 & 0,1 \\
\hline $\begin{array}{lllll}\text { Albacete } & \ldots & \ldots & \ldots & \ldots \\
\text { Ciudad } & \text { Real... } & \ldots & \ldots \\
\text { Cuenca } & \ldots & \ldots & \ldots & \ldots \\
\text { Guadalajara... } & \ldots & \ldots \\
\text { Toledo } & \ldots & \ldots & \ldots & \ldots \\
\end{array}$ & $\begin{array}{l}84,5 \\
90,5 \\
89,0 \\
83,4 \\
82,3\end{array}$ & $\begin{array}{l}6,4 \\
4,9 \\
6,6 \\
8,1 \\
8,4\end{array}$ & $\begin{array}{l}5,1 \\
3,0 \\
3,2 \\
4,5 \\
7,0\end{array}$ & $\begin{array}{l}2,7 \\
1,1 \\
0,8 \\
1,6 \\
1,5\end{array}$ & $\begin{array}{l}1,3 \\
0,5 \\
0,4 \\
2,1 \\
0,7\end{array}$ & $\begin{array}{l}- \\
\overline{0} \\
0,3 \\
0,1\end{array}$ \\
\hline $\begin{array}{rrrrr}\text { CATALUNA } & \ldots & \ldots & \ldots & \ldots \\
\text { Barcelona... } & \ldots & \ldots & \ldots \\
\text { Gerona } & \ldots & \ldots & \ldots & \ldots \\
\text { Lérida } & \ldots & \ldots & \ldots & \ldots \\
\text { Tarragona } & \ldots & \ldots & \ldots\end{array}$ & $\begin{array}{l}70,4 \\
66,4 \\
73,3 \\
85,6 \\
81,8\end{array}$ & $\begin{array}{r}12,3 \\
13,7 \\
11,1 \\
7,1 \\
8,1\end{array}$ & $\begin{array}{r}10,4 \\
11,9 \\
9,6 \\
4,9 \\
6,2\end{array}$ & $\begin{array}{l}3,3 \\
3,8 \\
3,3 \\
1,3 \\
2,0\end{array}$ & $\begin{array}{l}3,2 \\
3,8 \\
2,5 \\
1,0 \\
1,8\end{array}$ & $\begin{array}{l}0,4 \\
0,4 \\
0,2 \\
0,1 \\
0,1\end{array}$ \\
\hline $\begin{array}{rllll}\text { EXTREMADURA } & \ldots & \ldots \\
\text { Badajoz } & \ldots & \ldots & \ldots & \ldots \\
\text { Cáceres } & \ldots & \ldots & \ldots & \ldots\end{array}$ & $\begin{array}{l}91,5 \\
91,7 \\
91,1\end{array}$ & $\begin{array}{l}4,2 \\
4,3 \\
4,2\end{array}$ & $\begin{array}{l}3,0 \\
2,8 \\
3,4\end{array}$ & $\begin{array}{l}0,5 \\
0,5 \\
0,4\end{array}$ & $\begin{array}{l}0,6 \\
0,6 \\
0,7\end{array}$ & $\begin{array}{l}0,2 \\
0,2 \\
0,2\end{array}$ \\
\hline 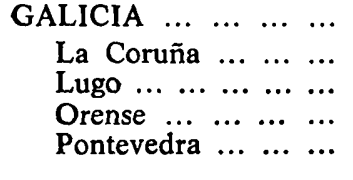 & $\begin{array}{l}84,2 \\
82,7 \\
91,8 \\
88,1 \\
77,6\end{array}$ & $\begin{array}{l}6,9 \\
7,3 \\
4,0 \\
6,1 \\
9,4\end{array}$ & $\begin{array}{l}5,6 \\
6,5 \\
2,8 \\
4,1 \\
7,5\end{array}$ & $\begin{array}{l}1,8 \\
1,8 \\
0,8 \\
0,9 \\
3,1\end{array}$ & $\begin{array}{l}1,4 \\
1,5 \\
0,6 \\
0,8 \\
2,1\end{array}$ & $\begin{array}{l}0,1 \\
0,2 \\
\overline{0,3}\end{array}$ \\
\hline 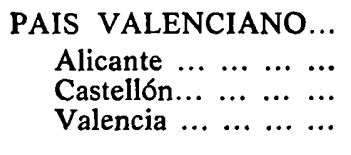 & $\begin{array}{l}70,2 \\
68,3 \\
77,3 \\
69,8\end{array}$ & $\begin{array}{r}13,1 \\
14,1 \\
7,6 \\
13,8\end{array}$ & $\begin{array}{r}11,7 \\
13,1 \\
9,5 \\
11,3\end{array}$ & $\begin{array}{l}3,0 \\
2,9 \\
3,4 \\
2,9\end{array}$ & $\begin{array}{l}1,8 \\
1,5 \\
2,1 \\
2,0\end{array}$ & $\begin{array}{l}0,2 \\
0,1 \\
0,1 \\
0,2\end{array}$ \\
\hline $\begin{array}{ccccc}\text { PAIS VASCO } & \ldots & \ldots & \ldots \\
\text { Alava } & \ldots & \ldots & \ldots & \ldots \\
\text { Guipúzcoa } & \ldots & \ldots & \ldots \\
\text { Vizcaya } & \ldots & \ldots & \ldots & \ldots\end{array}$ & $\begin{array}{l}61,5 \\
64,7 \\
58,1 \\
62,9\end{array}$ & $\begin{array}{l}14,2 \\
12,0 \\
15,8 \\
13,8\end{array}$ & $\begin{array}{l}12,6 \\
11,8 \\
13,5 \\
12,1\end{array}$ & $\begin{array}{l}5,4 \\
6,0 \\
6,0 \\
9,8\end{array}$ & $\begin{array}{l}5,4 \\
4,6 \\
5,8 \\
5,4\end{array}$ & $\begin{array}{l}0,9 \\
0,9 \\
0,8 \\
1,0\end{array}$ \\
\hline 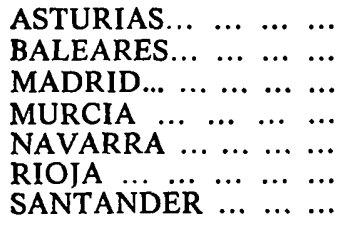 & $\begin{array}{l}78,9 \\
84,7 \\
67,0 \\
78,6 \\
70,1 \\
74,3 \\
74,5\end{array}$ & $\begin{array}{r}8,6 \\
7,7 \\
14,0 \\
9,7 \\
11,4 \\
10,0 \\
10,7\end{array}$ & $\begin{array}{r}7,3 \\
5,5 \\
1,8 \\
7,6 \\
10,7 \\
10,6 \\
8,0\end{array}$ & $\begin{array}{l}2,0 \\
1,3 \\
3,5 \\
1,8 \\
4,0 \\
3,2 \\
3,3\end{array}$ & $\begin{array}{l}2,4 \\
0,8 \\
3,2 \\
2,0 \\
3,2 \\
1,7 \\
2,6\end{array}$ & $\begin{array}{l}0,8 \\
0,5 \\
0,3 \\
0,6 \\
0,2 \\
0,9\end{array}$ \\
\hline $\begin{array}{llllll}\text { Ceuta } & \ldots & \ldots & \ldots & \ldots & \ldots \\
\text { Melilla } & \ldots & \ldots & \ldots & \ldots\end{array}$ & $\begin{array}{l}69,4 \\
84,6\end{array}$ & $\begin{array}{r}19,0 \\
6,3\end{array}$ & $\begin{array}{l}9,9 \\
4,9\end{array}$ & $\begin{array}{l}1,7 \\
4,2\end{array}$ & - & - \\
\hline
\end{tabular}


REVL-1981, núm. 210. BALLESTER ROS, IGNACIO. ALGUNAS CARACTERISTICAS DE LOS ESTA...

REVL-1981, núm. 210. BALLESTER ROS, IGNACIO. ALGUNAS CARACTERISTICAS DE LOS ESTA... 


\section{REVISTA}

DExitus

(9) DE LA

\section{JURISPRUDENCIA}


REVL-1981, núm. 210. BALLESTER ROS, IGNACIO. ALGUNAS CARACTERISTICAS DE LOS ESTA... 